\title{
Water quality index and fractal dimension analysis of water parameters
}

\author{
K. S. Parmar $\cdot$ R. Bhardwaj
}

Received: 23 January 2012/Revised: 27 March 2012/Accepted: 14 June 2012/Published online: 10 August 2012

(c) CEERS, IAU 2012

\begin{abstract}
Statistical analysis of water quality parameters were analyzed at Harike Lake on the confluence of Beas and Sutlej rivers of Punjab (India). Mean, median, mode, standard deviation, kurtosis, skewness, coefficient of variation, regression lines, correlation coefficient, Hurst exponent, fractal dimension and predictability index were estimated for each water parameter. Monthly variation of water quality index using month-wise and parameter-wise value of quality rating and actual value present in water sample was calculated and compared with World Health Organization/ Environmental Protection Agency standard value of these parameters. It was observed that Brownian time series behavior exists of potential of hydrogen with total dissolved solids, hardness, alkalinity, sulfate, chloride and conductance parameters; biochemical oxygen demand with total dissolved solids, hardness, alkalinity, sulfate, chloride, conductance and calcium parameters; dissolved oxygen with total dissolved solids, hardness, alkalinity, sulfate, chloride, conductance and calcium parameters; ferrous with total dissolved solids, hardness, alkalinity, sulfate, conductance and calcium parameters; chromium with total dissolved solids, hardness, alkalinity, sulfate, chloride, conductance and zinc parameters; zinc with total dissolved solids, hardness, sulfate, chloride, conductance and calcium parameters; fluoride with total dissolved solids, hardness, alkalinity, sulfate, chloride and conductance parameters; nitrate with total dissolved solids, sulfate and conductance parameters; nitrite with potential of hydrogen, total dissolved solids, hardness, alkalinity, sulfate, chloride, conductance and
\end{abstract}

\section{K. S. Parmar · R. Bhardwaj $(\bowtie)$}

Department of Mathematics, University School of Basic and Applied Sciences, Guru Gobind Singh Indraprastha University, Dwarka 110075, Delhi, India

e-mail: rashmib22@gmail.com calcium parameters. Also, using water quality index, it was observed that water of the lake was severely contaminated and became unfit for drinking and industrial use.

Keywords Statistical analysis - Hurst exponent . Fractal dimension $\cdot$ Predictability index

\section{Introduction}

Rivers are most important resources of water. Water is a valuable natural resource and natural water always contains dissolved and suspended substances of organic and mineral origin (Prasad and Narayana 2004). Sutlej and Beas rivers reach Harike Lake (Punjab, India) after crossing many states of India and effluents from different cities, towns and villages get mixed and make the river water polluted. Pollution in river water is continuously increasing due to urbanization, industrialization, etc. Many rivers are dying due to pollution which is an alarming signal (Jain et al. 2005; Phiri et al. 2005; Parmar et al. 2009). Water quality parameters and the effects of trace metals from industrial wastes, municipal sewage and agricultural runoff on river water quality have been investigated (Alam et al. 2007; Akoto and Adiyiah 2007). The analysis of the simultaneous effect of water pollution and eutrophication on the concentration of dissolved oxygen (DO) in a water body shows that the decrease in the concentration of DO is much more than when only a single effect is present on the water body, thus leading to more uncertainty about the survival of DOdependent species (Shukla et al. 2008). Water quality index (WQI) technique provides a single number that expresses the overall water quality at a certain location and time, based on several water quality parameters (Kumar and Dua 2009). Qualitative analysis and World Health Organization/ 
Environmental Protection Agency (WHO/EPA) water quality standards (WHO (1971) are used for calculating water quality indices.

Treatment of domestic wastewater using laboratory-scale hybrid upflow anaerobic sludge blanket (HUASB) reactor reduced the treatment cost significantly (Banu et al. 2007). The oxidation treatment system constructed under the riverbed of Nan-men Stream located at the Shin Chu City of Taiwan has been modeled such that it has significant efficiency (Juang et al. 2008). Trihalomethane compounds were determined in the drinking water samples that were collected from the selected consumption sites and treatment plants of both Okinawa and Samoa islands and it was observed that the chloroform and bromodichloromethane compound exceeded the level of Japan water quality and WHO standards (Imo et al. 2007). Water quality of watersheds is studied using hydrochemical data that mingle multiple linear regression and structural equation modeling (Chenini and Khemiri 2009; Chenini et al. 2008; Mousavi et al. 2008). Regression equations can be used to estimate constituent concentrations. Constituent concentrations can be used by water-quality managers for comparison of current water-quality conditions to water-quality standards. Examination of stream flow and physical properties of water that act as surrogates for constituents of interest also helps in the collection of waterquality samples (Vassilis et al. 2001; Psargaonkar et al. 2008; Joarder et al. 2008; Carlson and Ecker 2002; Korashey 2009). The statistical regression analysis of underground drinking water obtain from $\mathrm{IM}_{2}$ hand pumps at Moradabad, India was studied and observed that drinking water quality could be checked effectively by controlling the conductivity of water (Sinha and Kumar 2010).

The dispersion coefficient represents the rate of pollution and is an important parameter for air or water pollution modeling. In general, two- or one-dimensional dispersion coefficients are required for modeling. Fuzzy logic model based on Mamdani approach was developed to estimate the flow for poorly gauged mountainous basins. The stream and time coefficients were used as variables for modeling. The data were divided into training and testing phases. The model results were compared with the measured data. The comparison depends on seven statistical characteristics, four different error modes and the contour map method. Thus, the fuzzy model provides more accurate and reliable results (Toprak 2009; Toprak et al. 2004, 2009; Toprak and Savci 2007; Aksoy et al. 2004; Toprak and Cigizoglu 2008).

The Indian climate dynamics was studied using fractal dimensional analysis and analyzed time series data of three major dynamic components, i.e., temperature, pressure and precipitation. It has been observed that regional climate models would not be able to predict local climate as these deal with averaged quantities and the precipitation during the southwest monsoon is affected by temperature and pressure variability during the preceding winter. Time series can be modeled by a stochastic process possessing long range correlation (Rangarajan and Sant 2004; Rangarajan and Ding 2000; Movahed and Hermanisc 2008; Kahya and Kalayci 2004). Hurst parameter for long rangedependent processes using wavelet technique provides the asymptotic linear relationship of the basis for construction of an estimator (Park and Park 2009).

In this study, statistical analysis, regression equations, Hurst exponent, fractal dimension, predictability index and water quality index of water parameters were estimated at the confluence of Sutlej and Beas rivers at Harike Lake, Punjab. The river map of India is shown in Fig. 1.

\section{Methodology}

Chemical analysis

The water samples were collected from Harike Lake (on the confluence of Sutlej and Beas rivers) once in a month for a year. These samples were collected in 2-L bottles, properly rinsed with $8 \mathrm{~mL}$ of $\mathrm{HNO}_{3}$ and followed by repeated washing with distilled water. Using the standard methods and procedures of sampling and estimation as prescribed in APHA (1995), the 16 water quality physicochemical parameters including 6 cations, 5 anions besides dissolved oxide (DO), biochemical oxygen demand (BOD), total dissolved solids (TDS), potential of hydrogen $(\mathrm{pH})$ and conductivity were measured.

\section{Water quality index (WQI)}

The quality rating $Q_{n}$ is defined as:

$Q_{n}=100\left[\frac{v_{n}-v_{i}}{v_{s}-v_{i}}\right]$

where $v_{n}$ is the actual value of each water parameter present in the water sample. $v_{i}$ is the ideal value of each water parameter ( 0 for all parameters except for $\mathrm{pH}$ and DO whichare $7.0 \mathrm{mg} / \mathrm{L}$ and $14.6 \mathrm{mg} / \mathrm{L}$, respectively). $v_{s}=$ recommended WHO/EPA standard of each parameter (Parmar et al. 2009).

Unit weights $W_{n}$ for each water parameter are inversely proportional to $S_{n}$ (water quality standard adopted worldwide as prescribed by $\mathrm{WHO} / \mathrm{EPA})$. i.e.,

$W_{n}=\frac{k}{S_{n}}$

where $k$ is a constant of proportionality. Let

$\sum_{n=1}^{16} W_{n}=1$

and sub-indices (SI) be given by 


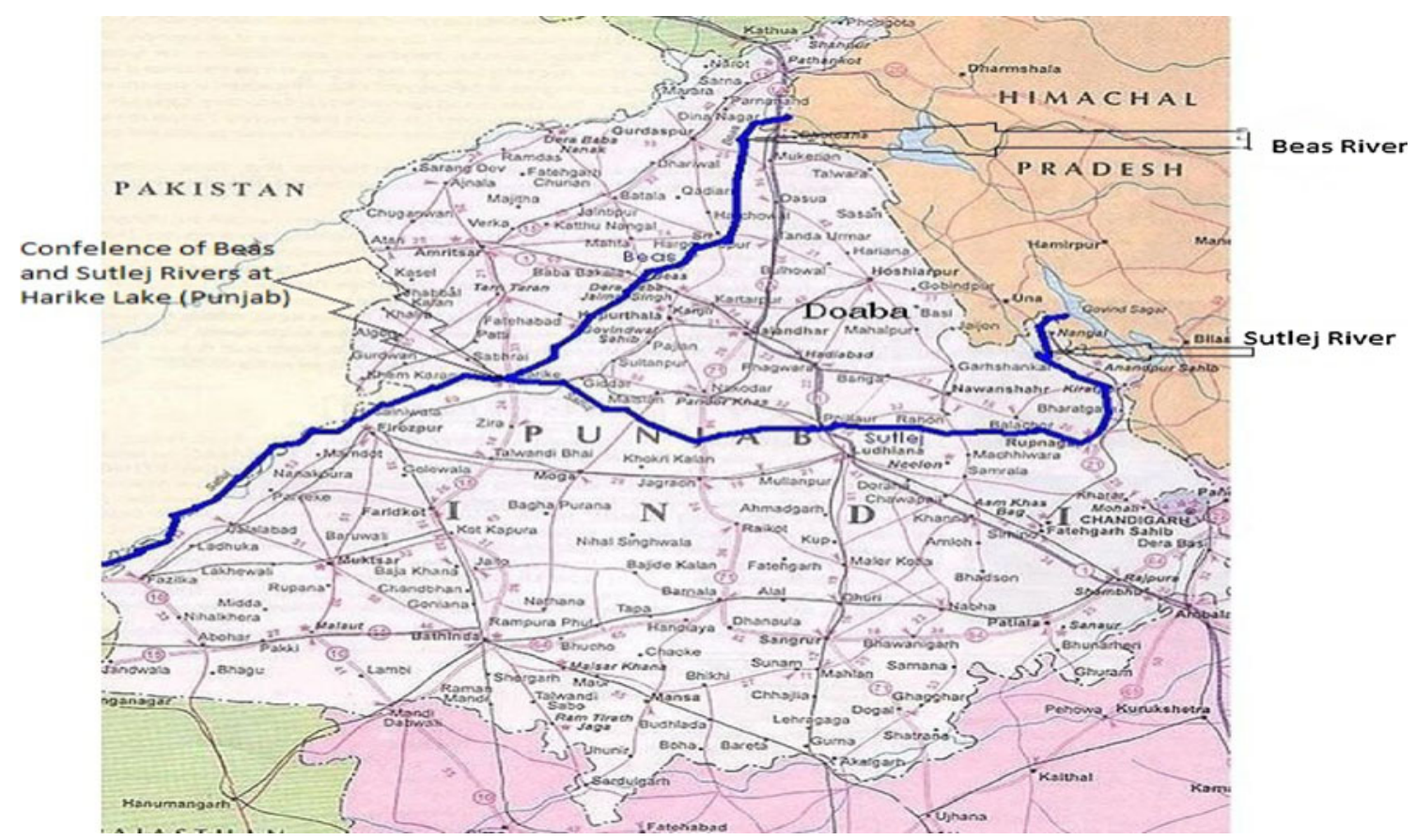

Fig. 1 Confluence of Sutlej and Beas rivers at Harike Lake (Punjab)

$(\mathrm{S} \mathrm{I})_{n}=\left(Q_{n}\right)^{W_{n}}$

The overall WQI is defined (by taking geometric mean of sub indices) as:

$$
\begin{aligned}
\mathrm{WQI} & =\prod_{n=1}^{16}(\mathrm{SI})_{n}=\prod_{n=1}^{16}\left(Q_{n}\right)^{W_{n}} \\
& =\text { anti } \log _{10}\left[\sum_{n=1}^{16} W_{n} \log _{10} Q_{n}\right]
\end{aligned}
$$

In order to assess the extent of contamination or the quality of drinking water, the following assumptions are made:

WQI $<50$ : fit for consumption.

$50<$ WQI $<80$ : moderately contaminated.

$80<$ WQI $<100$ : excessively contaminated.

WQI > 100: severely contaminated (Kumar and Dua 2009).

\section{Statistical analysis}

Statistical analysis is used to calculate mean, median, mode, standard deviation, kurtosis, skewness and coefficient of variation. Mean explains the average value. Median gives the middle values of an ordered sequence or positional average. Mode is defined as the value which occurs the maximum number of times that has the maximum frequency. Standard deviation gives the measure of "spread" or "variability" of the sample. Kurtosis refers to the degree of flatness or peakedness in the region about the mode of a frequency curve. Skewness describes the symmetry of data. Coefficient of variation gives the relative measure of the sample (Rangarajan 1997).

\section{Regression analysis}

It is a technique used for modeling and analyzing the variables present in a sample. Regression analysis helps in understanding the variation in value of the dependent variable as independent variables are varied, while the other independent variables are held fixed. Regression line of $Y$ (dependent variable) on $X$ (independent variable) is defined as

$$
Y=b_{y x} X+C
$$

(Chenini and Khemiri 2009) where $C$ is a constant of integration,

$b_{y x}=$ regression coefficient $=r \times \frac{\sigma_{y}}{\sigma_{x}}$

$r=$ Correlation coefficient

$$
=\frac{E(X Y)-E(X) E(Y)}{\sqrt{\left(E\left(X^{2}\right)-E(X)^{2}\right)\left(E\left(Y^{2}\right)-E(Y)^{2}\right)}}=\frac{\operatorname{cov}(\mathrm{X}, \mathrm{Y})}{\sigma_{X} \sigma_{Y}}
$$

$\sigma_{Y}, \sigma_{X}$ are standard deviation of variables $Y$ and $X$, respectively, and $E(X), E(Y), E(X Y)$ are the expected value of variables $X, Y$ and $X Y$, respectively. 


\section{Hurst exponent $(H)$}

It refers to the index of dependence. It quantifies the relative tendency of a time series either to regress strongly to the mean or to cluster in a direction. The value of the Hurst exponent ranges between 0 and 1 . A value of 0.5 indicates a true random walk (a Brownian time series). In a random walk, there is no correlation between any element and a future element. A Hurst exponent value $H, 0.5<H<1$ indicates "persistent behavior" (a positive autocorrelation). If there is an increase from time step $t_{i-1}$ to $t_{i}$, there will probably be an increase from $t_{i}$ to $t_{i+1}$. The same is true of decreases, where a decrease will tend to follow a decrease. A Hurst exponent value, $H 0<H<0.5$, will exist for a time series with "anti-persistent behavior" (or negative autocorrelation). Here, an increase will tend to be followed by a decrease or decrease will be followed by an increase. This behavior is sometimes called "mean reversion".

$H=\left|\frac{b_{y x}-1}{2}\right|$

(Rangarajan and Sant 2004). Also, Hurst exponent can be calculated using power law decay

$p(k)=C k^{-a}$

(Rangarajan and Ding 2000) where $C$ is a constant and $p(k)$ is the autocorrelation function with lag $k$. The Hurst exponent is related to the exponent alpha in the equation by the relation

$H=1-\frac{\alpha}{2}$

\section{Fractal dimension $(D)$}

It is a statistical quantity that gives an indication of how completely a fractal appears to fill space, as one zooms down to finer and finer scales.

$D=2-H$

(Rangarajan and Sant 2004).

Also, fractal dimension is calculated from the Haussdorf dimension. The Haussdorf dimension, $D_{H}$, in a metric space is defined as

$D_{H}=-\lim _{\varepsilon \rightarrow 0} \frac{\ln [N(\varepsilon)]}{\ln \varepsilon}$

where $N(\varepsilon)$ is the number of open balls of a radius $\varepsilon$ needed to cover the entire set. An open ball with center $P$ and radius $\varepsilon$ in a metric space with metric $\mathrm{d}$ is defined as a set of all points $x$ such that $\mathrm{d}(P, x)<\varepsilon$.

\section{Predictability index $(P I)$}

It describes the behavior of the time series

$P I=2|D-1.5|$
(Rangarajan 1997). PI value increases when $D$ value becomes less than or greater than 1.5. In the former case, persistence behavior is observed, while in the latter, an anti-persistence. If one of these indices comes close to 0 , then the corresponding process approximates the Brownian motion and is therefore unpredictable.

\section{Results and discussion}

Using Eqs. (1) and (2), the estimated quality rating $Q_{n}$, actual value $\left(v_{n}\right)$ along with WHO/EPA standards $\left(v_{s}\right)$ and assigned unit weights $\left(W_{n}\right)$ of 16 physico-chemical parameters are calculated and shown in Table 1. Using Eq. (3), the monthly value of water quality index (WQI) was calculated and shown in Table 2. Using values from Table 2, graphs are plotted as in Fig. 2. It was observed that WQI calculated values were exceptionally high compared with the prescribed limits. Due to heavy rainfall during July, August, September, December and January, dilution occurs in lake water and a minimum value of WQI is observed in rainy months. In the months from July to September, rains occur due to monsoons, but in winter (December-February) rains occur due to typhoons from the Persian Gulf. In the summer season, due to high temperature (up to $46^{\circ} \mathrm{C}$ ), high rate of evaporation takes place and the concentration of contaminants increases during this season. In general, it was observed that lake water was severely contaminated and became unfit for drinking and industrial use.

Table 3 shows the mean, median, mode, standard deviation, skewness, kurtosis and coefficient of variation of the 16 water quality parameters at Harike Lake, which is also plotted in Fig. 3. Using Eqs. (4, 9), regression equations, coefficient of correlation, Hurst exponent, fractal dimension and predictability index value of water parameters at Harike Lake were calculated and shown in Table 4. From Tables 3 and 4, the following results were observed.

$\mathrm{pH}$

Average value, positional average and mode of $\mathrm{pH}$ were 8.37, 8.375 and 8.383, respectively. These values are approximate to 8.37 ; thus, the data indicate normal behavior. Standard deviation (SD) is 0.121 and skewness is approximate to 0 , thus $\mathrm{pH}$ is symmetrical and values are very close to each other. The curve is platykurtic, as kurtosis is less than 3. $\mathrm{pH}$ shows Brownian time series (true random walk) behavior with TDS, hardness, alkalinity, sulfate, chloride and conductance parameters, persistent behavior with BOD, DO, chromium $(\mathrm{Cr})$ 
Table 1 Month-wise and parameter-wise values of $v_{n}$ and $Q_{n}$ with WHO/EPA standards $v_{s}$ in mg/L and assigned unit weights of water parameters at Harike Lake

\begin{tabular}{|c|c|c|c|c|c|c|c|}
\hline Parameters & Months & $v_{n}(\mathrm{mg} / \mathrm{L})$ & $\begin{array}{l}Q_{n} \\
(\mathrm{mg} / \mathrm{L})\end{array}$ & Parameters & Months & $v_{n}(\mathrm{mg} / \mathrm{L})$ & $\begin{array}{l}Q_{n} \\
(\mathrm{mg} / \mathrm{L})\end{array}$ \\
\hline $\mathrm{pH}$ & April & 8.3 & 130 & DO & April & 9.45 & 53.64 \\
\hline \multirow{2}{*}{$\begin{array}{l}\text { WHO/EPA Standard } v_{s} \\
=8.00\end{array}$} & May & 8.3 & 130 & WHO/EPA Standard $v_{s}=5$ & May & 9.6 & 52.083 \\
\hline & June & 8.4 & 140 & \multirow{10}{*}{$\begin{array}{l}\text { Assigned unit weight } \\
\left(W_{n}\right)=0.008219\end{array}$} & June & 9.5 & 53.125 \\
\hline \multirow{9}{*}{$\begin{array}{l}\text { Assigned unit weight } \\
\left(W_{n}\right)=0.0051373\end{array}$} & July & 8.4 & 140 & & July & 9.4 & 54.166 \\
\hline & August & 8.2 & 120 & & August & 9.64 & 51.66 \\
\hline & September & 8.2 & 120 & & September & 9.3 & 55.2 \\
\hline & October & 8.3 & 130 & & October & 9.1 & 57.29 \\
\hline & November & 8.5 & 150 & & November & 9.15 & 56.77 \\
\hline & December & 8.6 & 160 & & December & 9.25 & 55.72 \\
\hline & January & 8.5 & 150 & & January & 9.2 & 56.25 \\
\hline & February & 8.4 & 140 & & February & 9.31 & 55.2 \\
\hline & March & 8.35 & 135 & & March & 9.35 & 54.68 \\
\hline TDS & April & 300 & 60 & $\mathrm{Ca}$ & April & 28.12 & 28.12 \\
\hline WHO/EPA Standard $v_{s}=500$ & May & 460 & 92 & WHO/EPA Standard $v_{s}=100$ & May & 34.64 & 34.64 \\
\hline \multirow{10}{*}{$\begin{array}{l}\text { Assigned unit weight } \\
\left(W_{n}\right)=0.0000822\end{array}$} & June & 550 & 110 & \multirow{10}{*}{$\begin{array}{l}\text { Assigned unit weight } \\
\left(W_{n}\right)=0.0004099\end{array}$} & June & 32.16 & 32.16 \\
\hline & July & 580 & 116 & & July & 31.43 & 31.43 \\
\hline & August & 412 & 82.4 & & August & 21.3 & 21.3 \\
\hline & September & 320 & 64 & & September & 22.64 & 22.64 \\
\hline & October & 380 & 76 & & October & 24.03 & 24.03 \\
\hline & November & 520 & 104 & & November & 33.64 & 33.64 \\
\hline & December & 530 & 106 & & December & 30.23 & 30.23 \\
\hline & January & 510 & 102 & & January & 33.43 & 33.43 \\
\hline & February & 456 & 91.2 & & February & 35.09 & 35.09 \\
\hline & March & 330 & 66 & & March & 26 & 26 \\
\hline Hardness & April & 180 & 180 & $\mathrm{Fe}$ & April & 0.7 & 140 \\
\hline WHO/EPA Standard $v_{s}=100$ & May & 197 & 197 & WHO/EPA Standard $v_{s}=0.5$ & May & 0.32 & 64 \\
\hline \multirow{10}{*}{$\begin{array}{l}\text { Assigned unit weight } \\
\left(W_{n}\right)=0.0004099\end{array}$} & June & 206 & 206 & \multirow{10}{*}{$\begin{array}{l}\text { Assigned unit weight } \\
\left(W_{n}\right)=0.082198\end{array}$} & June & 0.28 & 56 \\
\hline & July & 210 & 210 & & July & 0.06 & 12 \\
\hline & August & 188 & 188 & & August & 0.2 & 40 \\
\hline & September & 165 & 165 & & September & 0.1 & 20 \\
\hline & October & 170 & 170 & & October & 0.12 & 24 \\
\hline & November & 170 & 170 & & November & 1.1 & 220 \\
\hline & December & 240 & 240 & & December & 1.16 & 232 \\
\hline & January & 240 & 240 & & January & 1.05 & 210 \\
\hline & February & 220 & 220 & & February & 0.8 & 160 \\
\hline & March & 190 & 190 & & March & 0.6 & 120 \\
\hline Alkalinity & April & 90 & 90 & $\mathrm{Cr}$ & April & 0.5 & 1,000 \\
\hline WHO/EPA Standard $v_{s}=100$ & May & 70 & 70 & \multirow{11}{*}{$\begin{array}{l}\text { WHO/EPA Standard } \\
v_{s}=0.05 \\
\text { Assigned unit weight } \\
\left(W_{n}\right)=0.82198\end{array}$} & May & 0.76 & 1,520 \\
\hline \multirow{10}{*}{$\begin{array}{l}\text { Assigned unit weight } \\
\left(W_{n}\right)=0.0004099\end{array}$} & June & 102 & 102 & & June & 0.95 & 1,900 \\
\hline & July & 110 & 110 & & July & 0.55 & 1,100 \\
\hline & August & 86.66 & 86.66 & & August & 0.29 & 580 \\
\hline & September & 90 & 90 & & September & 0.42 & 840 \\
\hline & October & 100 & 100 & & October & 0.9 & 1,800 \\
\hline & November & 125 & 125 & & November & 0.4 & 800 \\
\hline & December & 120 & 120 & & December & 0.17 & 340 \\
\hline & January & 110 & 110 & & January & 0.49 & 980 \\
\hline & February & 106 & 106 & & February & 0.61 & 1,220 \\
\hline & March & 100 & 100 & & March & 0.81 & 1,620 \\
\hline
\end{tabular}


Table 1 continued

\begin{tabular}{|c|c|c|c|c|c|c|c|}
\hline Parameters & Months & $v_{n}(\mathrm{mg} / \mathrm{L})$ & $\begin{array}{l}Q_{n} \\
(\mathrm{mg} / \mathrm{L})\end{array}$ & Parameters & Months & $v_{n}(\mathrm{mg} / \mathrm{L})$ & $\begin{array}{l}Q_{n} \\
(\mathrm{mg} / \mathrm{L})\end{array}$ \\
\hline Sulfate & April & 461.4 & 230.73 & $\mathrm{Zn}$ & April & 2.8 & 56 \\
\hline WHO/EPA Standard $v_{s}=200$ & May & 561.4 & 280.7 & WHO/EPA Standard $v_{s}=5$ & May & 4 & 80 \\
\hline \multirow{10}{*}{$\begin{array}{l}\text { Assigned unit weight } \\
\left(W_{n}\right)=0.0002054\end{array}$} & June & 621.2 & 310.65 & \multirow{10}{*}{$\begin{array}{l}\text { Assigned unit } \\
\text { weight }\left(W_{n}\right)=0.00082198\end{array}$} & June & 2.6 & 52 \\
\hline & July & 576.8 & 288.4 & & July & 2 & 40 \\
\hline & August & 407.3 & 203.65 & & August & 4.1 & 82 \\
\hline & September & 360.9 & 180.45 & & September & 2.2 & 44 \\
\hline & October & 462.7 & 231.35 & & October & 2.5 & 50 \\
\hline & November & 668.7 & 334.35 & & November & 2.7 & 54 \\
\hline & December & 678.7 & 339.35 & & December & 2.5 & 50 \\
\hline & January & 660.7 & 330.35 & & January & 2.5 & 50 \\
\hline & February & 500 & 250 & & February & 2.6 & 52 \\
\hline & March & 550 & 225 & & March & 2.7 & 54 \\
\hline Chloride & April & 30.5 & 15.25 & $\mathrm{Fl}$ & April & 1.4 & 93.33 \\
\hline WHO/EPA Standard $v_{s}=200$ & May & 30 & 15 & WHO/EPA Standard $v_{s}=1.5$ & May & 1.63 & 108.66 \\
\hline \multirow{10}{*}{$\begin{array}{l}\text { Assigned unit weight } \\
\left(W_{n}\right)=0.0002054\end{array}$} & June & 36.6 & 18.3 & \multirow{10}{*}{$\begin{array}{l}\text { Assigned unit weight } \\
\left(W_{n}\right)=0.027399\end{array}$} & June & 1.72 & 114.66 \\
\hline & July & 35 & 17.5 & & July & 1.7 & 113.33 \\
\hline & August & 30.8 & 15.4 & & August & 1.18 & 78.66 \\
\hline & September & 26 & 13 & & September & 0.74 & 49.33 \\
\hline & October & 27 & 13.5 & & October & 1.28 & 85.33 \\
\hline & November & 35 & 17.5 & & November & 1.18 & 78.66 \\
\hline & December & 45 & 22.5 & & December & 1.12 & 74.66 \\
\hline & January & 55 & 27.5 & & January & 1.05 & 70 \\
\hline & February & 45 & 22.5 & & February & 1.2 & 80 \\
\hline & March & 30 & 15 & & March & 1.3 & 86.66 \\
\hline Conductance & April & 225 & 56.25 & Nitrate & April & 4.27 & 42.7 \\
\hline WHO/EPA Standard $v_{s}=400$ & May & 385 & 96.25 & WHO/EPA Standard $v_{s}=10$ & May & 4.5 & 45 \\
\hline \multirow{10}{*}{$\begin{array}{l}\text { Assigned unit weight } \\
\left(W_{n}\right)=0.0001027\end{array}$} & June & 397 & 99.25 & \multirow{10}{*}{$\begin{array}{l}\text { Assigned unit weight } \\
\left(W_{n}\right)=0.004109\end{array}$} & June & 5.25 & 52.5 \\
\hline & July & 424 & 106 & & July & 5 & 50 \\
\hline & August & 344 & 86 & & August & 3.78 & 37.8 \\
\hline & September & 230 & 57.5 & & September & 3.5 & 35 \\
\hline & October & 260 & 65 & & October & 3.8 & 38 \\
\hline & November & 420 & 105 & & November & 5.85 & 58.5 \\
\hline & December & 440 & 110 & & December & 5.95 & 59.5 \\
\hline & January & 430 & 107.5 & & January & 5.5 & 55 \\
\hline & February & 375 & 93.75 & & February & 5.12 & 51.2 \\
\hline & March & 250 & 62.5 & & March & 4.46 & 44.6 \\
\hline BOD & April & 4.65 & 77.5 & Nitrite & April & 0.6 & 60 \\
\hline WHO/EPA Standard $v_{s}=6$ & May & 4.3 & 71.666 & WHO/EPA Standard $v_{s}=1$ & May & 0.35 & 35 \\
\hline \multirow{10}{*}{$\begin{array}{l}\text { Assigned unit weight } \\
\left(W_{n}\right)=0.006849\end{array}$} & June & 4.4 & 73.33 & \multirow{10}{*}{$\begin{array}{l}\text { Assigned unit weight } \\
\left(W_{n}\right)=0.041099\end{array}$} & June & 0.52 & 52 \\
\hline & July & 4.6 & 76.66 & & July & 0.55 & 55 \\
\hline & August & 4.3 & 71.66 & & August & 0.23 & 23 \\
\hline & September & 4.8 & 80 & & September & 0.8 & 80 \\
\hline & October & 4.9 & 81.66 & & October & 1.4 & 140 \\
\hline & November & 4.65 & 77.55 & & November & 1.25 & 125 \\
\hline & December & 4.55 & 75.83 & & December & 1.36 & 136 \\
\hline & January & 4.4 & 73.33 & & January & 1.2 & 120 \\
\hline & February & 4.5 & 75 & & February & 0.9 & 90 \\
\hline & March & 4.55 & 75.83 & & March & 0.8 & 80 \\
\hline
\end{tabular}


and zinc $(\mathrm{Zn})$, and anti-persistent behavior with calcium $(\mathrm{Ca})$, ferrous $(\mathrm{Fe})$, fluoride $(\mathrm{Fl})$, nitrate and nitrite parameters.

Table 2 Monthly value of water quality index at Harike Lake

\begin{tabular}{lc}
\hline Months & WQI values \\
\hline April & 659.065735 \\
May & 856.235719 \\
June & 1036.841797 \\
July & 584.124873 \\
Aug & 363.032166 \\
Sep & 483.34903 \\
Oct & 954.959595 \\
Nov & 585.756836 \\
Dec & 291.820343 \\
Jan & 685.687622 \\
Feb & 792.799255 \\
March & 977.38855 \\
\hline
\end{tabular}

\section{TDS}

Mean, median and mode values are different; thus, the curve does not follow normal behavior. Standard deviation value is high (96.226), thus the values of TDS are not close to each other. It is negatively skewed and the curve is platykurtic. TDS has persistent behavior with hardness and anti-persistent behavior with sulfate and conductance.

\section{Hardness}

Mean and median values are approximately equal, but mode value is different. So, it does not exhibit normal behavior. Standard deviation value (25.877) suggests that data are spread out and the curve is platykurtic. Hardness has persistent behavior with chloride, DO and $\mathrm{Ca}$, and antipersistent behavior with TDS, alkalinity, sulfate and conductance parameters.

Fig. 2 Monthly values of water quality index (WQI) at Harike Lake

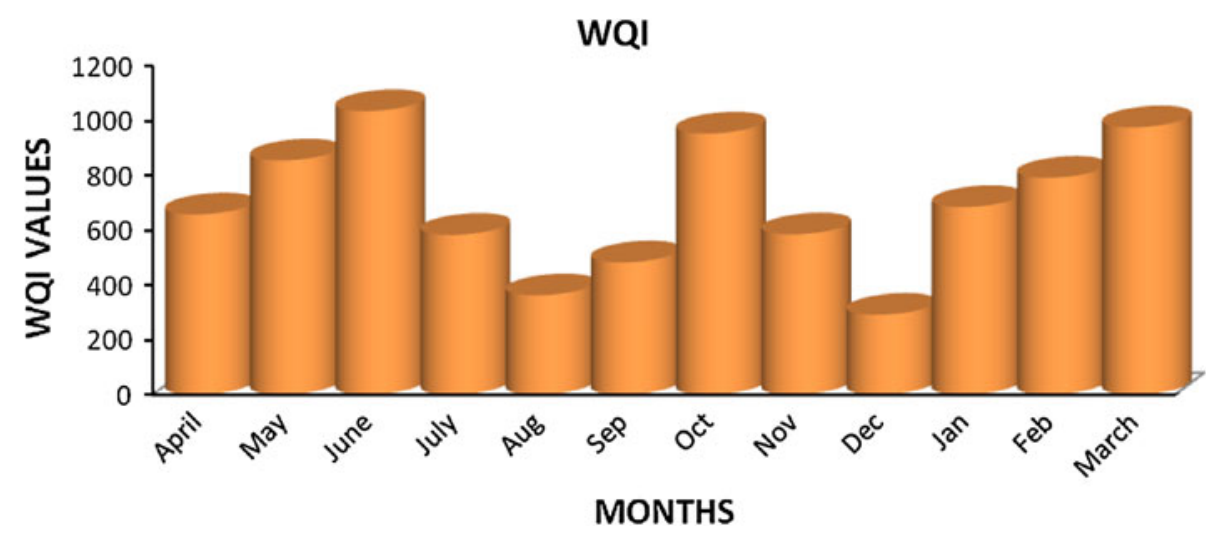

Table 3 Statistical analysis of water parameters at Harike Lake

\begin{tabular}{lccccccc}
\hline Parameters & Mean & Median & Mode & SD & Skewness & Kurtosis & Coefficient of variation \\
\hline pH & 8.37 & 8.375 & 8.383 & 0.121 & 0.327 & -0.344 & 0.015 \\
TDS & 445.667 & 458 & 482.667 & 96.226 & -0.257 & -1.385 & 0.216 \\
Hardness & 198 & 193.5 & 184.5 & 25.877 & 0.465 & -0.865 & 0.131 \\
Alkalinity & 100.805 & 101 & 101.39 & 15.181 & -0.363 & 0.302 & 0.151 \\
Sulfate & 542.4833 & 555.7 & 582.133 & 105.417 & -0.265 & -1.046 & 0.194 \\
Chloride & 35.492 & 32.9 & 27.717 & 8.699 & 1.179 & 0.847 & 0.245 \\
Conductance & 348.333 & 380 & 443.333 & 83.7 & -0.535 & -1.58 & 0.24 \\
BOD & 4.55 & 4.55 & 4.55 & 0.186 & 0.382 & -0.343 & 0.04 \\
DO & 9.354 & 9.33 & 9.282 & 0.171 & 0.27 & -0.789 & 0.018 \\
Ca & 29.392 & 30.83 & 33.705 & 4.86 & -0.504 & -1.263 & 0.165 \\
Fe & 0.541 & 0.46 & 0.298 & 0.414 & 0.348 & -1.569 & 0.766 \\
Cr & 0.571 & 0.525 & 0.433 & 0.243 & 0.113 & -0.856 & 0.426 \\
Zn & 2.767 & 2.6 & 2.267 & 0.639 & 1.485 & 1.592 & 0.231 \\
Fl & 1.292 & 1.24 & 1.137 & 0.287 & -0.0183 & -0.004 & 0.222 \\
Nitrate & 4.748 & 4.75 & 4.753 & 0.823 & -0.032 & -1.232 & 0.173 \\
Nitrite & 0.83 & 0.8 & 0.74 & 0.398 & 0.118 & -1.303 & 0.479 \\
\hline
\end{tabular}


Fig. 3 Graphical representation of statistical analysis of water parameters at Harike Lake
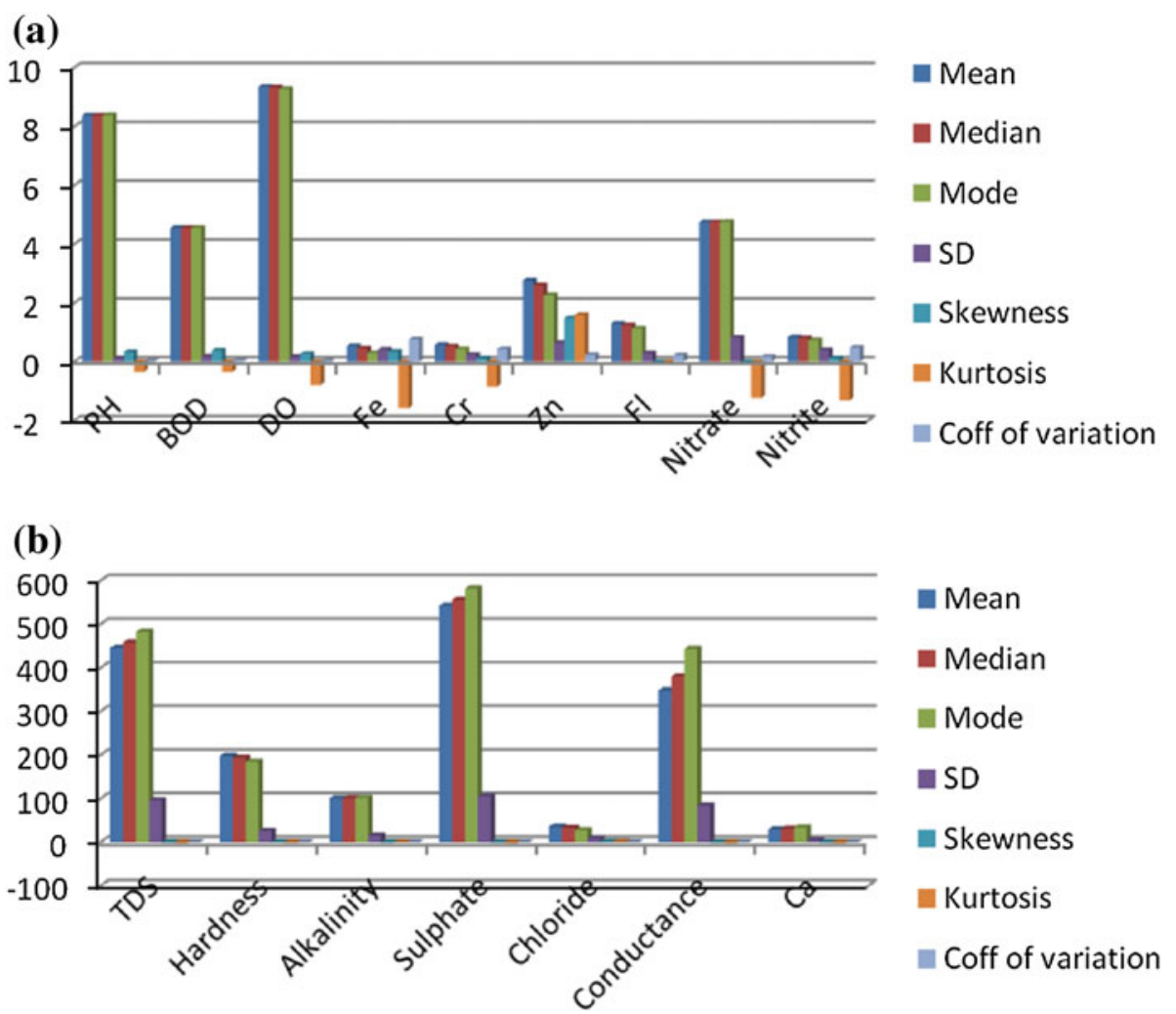

Alkalinity

Average, median and mode values are approximately equal and thus the data exhibit normal behavior. Standard deviation (15.181) suggests that the data are not close to each other. The skewness value $(-0.363)$ is approximately equal to 0 , thus the curve is symmetrical and platykurtic. Alkalinity has anti-persistent behavior with TDS, hardness, sulfate, chloride, conductance and $\mathrm{Ca}$.

\section{Sulfate}

Mean, median and mode values are approximately equal. Standard deviation (105.417) suggests that sample data are not close to each other. The skewness value is approximately equal to 0 , thus the curve is symmetrical and platykurtic. Sulfate has persistent behavior with hardness and anti-persistent behavior with TDS and conductance.

\section{Chloride}

Average, median and mode values are approximately equal, and thus the data show normal behavior. Standard deviation value (8.699) shows that the sample points are not close to each other. Skewness value suggests that the curve is symmetrical and the kurtosis value is less than 3 . Thus, the curve is platykurtic. Chloride has anti-persistent behavior with TDS, hardness, alkalinity, sulfate and conductance.
Conductance

Mean, median and mode values are different, and thus the data do not show normal behavior. Standard deviation value (83.7) suggests that data are spread. The curve is negatively skewed and platykurtic. Conductance has persistent behavior with hardness, alkalinity and $\mathrm{Zn}$ and antipersistent behavior with TDS and sulfate.

\section{BOD}

Average, median and mode values are the same, and thus the data show normal behavior. Standard deviation value (0.186) explains that the sample data are not spread. The curve is skewed and platykurtic. BOD has Brownian time series (True random walk) behavior with TDS, hardness, alkalinity, sulfate, chloride, conductance and Ca parameters. BOD has persistent behavior with $\mathrm{pH}, \mathrm{DO}, \mathrm{Fe}, \mathrm{Zn}, \mathrm{Fl}$ and nitrate, and anti-persistent behavior with $\mathrm{Cr}$ and nitrite parameters.

\section{DO}

Mean and median values are approximately equal. Standard deviation (0.171) suggests that sample data are very close to each other. The skewness value is approximately equal to 0 , and thus the curve is symmetrical and platykurtic. DO has Brownian time series (true random walk) 
Table 4 Regression equations, coefficient of correlation, Hurst exponent, fractal dimension and predictability index between water parameters at Harike Lake

\begin{tabular}{|c|c|c|c|c|c|c|}
\hline Y & Parameters $(X)$ & Regression equation & $r^{2}$ & $H$ & $D$ (Fractal) & PI \\
\hline \multirow[t]{15}{*}{$\mathrm{pH}$} & TDS & $Y=0.0009 X+7.9854$ & 0.4694 & 0.5 & 1.5 & 0 \\
\hline & Hardness & $Y=0.0032 X+7.7391$ & 0.462 & 0.5 & 1.5 & 0 \\
\hline & Alkalinity & $Y=0.006 X+7.7168$ & 0.6577 & 0.5 & 1.5 & 0 \\
\hline & Sulfate & $Y=0.0011 X+7.799$ & 0.837 & 0.499 & 1.50055 & 0.0011 \\
\hline & Chloride & $Y=0.0106 X+7.9952$ & 0.5747 & 0.5 & 1.5 & 0 \\
\hline & Conductance & $Y=0.001 X+8.0064$ & 0.5196 & 0.499 & 1.5005 & 0.001 \\
\hline & BOD & $Y=-0.0658 X+8.6702$ & 0.0101 & 0.533 & 1.4671 & 0.0658 \\
\hline & DO & $Y=-0.348 X+11.626$ & 0.2392 & 0.674 & 1.326 & 0.348 \\
\hline & $\mathrm{Ca}$ & $Y=0.0164 X+7.8874$ & 0.4331 & 0.492 & 1.5082 & 0.0164 \\
\hline & $\mathrm{Fe}$ & $Y=0.2291 X+8.2469$ & 0.6103 & 0.386 & 1.61455 & 0.2291 \\
\hline & $\mathrm{Cr}$ & $Y=-0.1165 X+8.4373$ & 0.0543 & 0.558 & 1.44175 & 0.1165 \\
\hline & $\mathrm{Zn}$ & $Y=-0.075 X+8.5784$ & 0.1557 & 0.537 & 1.4625 & 0.075 \\
\hline & $\mathrm{Fl}$ & $Y=0.0212 X+8.3435$ & 0.0025 & 0.489 & 1.5106 & 0.0212 \\
\hline & Nitrate & $Y=0.1412 X+7.7006$ & 0.9141 & 0.429 & 1.5706 & 0.1412 \\
\hline & Nitrite & $Y=0.1873 X+8.2154$ & 0.3767 & 0.406 & 1.59365 & 0.1873 \\
\hline \multirow[t]{3}{*}{ TDS } & Hardness & $Y=2.2661 X-3.0186$ & 0.3714 & 0.63305 & 1.36695 & 0.2661 \\
\hline & Sulfate & $Y=0.6927 X+69.869$ & 0.5759 & 0.15365 & 1.84635 & 0.6927 \\
\hline & Conductance & $Y=1.0967 X+63.664$ & 0.9099 & 0.04835 & 1.95165 & 0.9033 \\
\hline \multirow[t]{7}{*}{ Hardness } & TDS & $Y=1.1639 X+124.96$ & 0.3714 & 0.08195 & 1.91805 & 0.8361 \\
\hline & Alkalinity & $Y=0.5922 X+138.3$ & 0.1207 & 0.2039 & 1.7961 & 0.5922 \\
\hline & Sulfate & $Y=0.149 X+117.15$ & 0.3686 & 0.4255 & 1.5745 & 0.149 \\
\hline & Chloride & $Y=2.6206 X+104.99$ & 0.7762 & 0.8103 & 1.1897 & 0.6206 \\
\hline & Conductance & $Y=0.2147 X+123.2$ & 0.4825 & 0.39265 & 1.60735 & 0.2147 \\
\hline & DO & $Y=-0.8737 X+206.17$ & $3.00 \mathrm{E}-05$ & 0.93685 & 1.06315 & 0.8737 \\
\hline & $\mathrm{Ca}$ & $Y=2.9115 X+112.43$ & 0.299 & 0.95575 & 1.04425 & 0.9115 \\
\hline \multirow[t]{6}{*}{ Alkalinity } & TDS & $Y=0.0838 X+63.461$ & 0.2821 & 0.4581 & 1.5419 & 0.0838 \\
\hline & Hardness & $Y=0.2038 X+60.447$ & 0.1207 & 0.3981 & 1.6019 & 0.2038 \\
\hline & Sulfate & $Y=0.0914 X+51.234$ & 0.4026 & 0.4543 & 1.5457 & 0.0914 \\
\hline & Chloride & $Y=0.9603 X+66.723$ & 0.3028 & 0.01985 & 1.98015 & 0.9603 \\
\hline & Conductance & $Y=0.0886 X+69.932$ & 0.2388 & 0.4557 & 1.5443 & 0.0886 \\
\hline & $\mathrm{Ca}$ & $Y=0.9783 X+72.05$ & 0.0981 & 0.01085 & 1.98915 & 0.9783 \\
\hline \multirow[t]{3}{*}{ Sulfate } & TDS & $Y=0.8314 X+171.96$ & 0.5759 & 0.0843 & 1.9157 & 0.8314 \\
\hline & Hardness & $Y=2.4732 X+52.792$ & 0.3686 & 0.7366 & 1.2634 & 0.4732 \\
\hline & Conductance & $Y=0.9805 X+200.96$ & 0.606 & 0.00975 & 1.99025 & 0.9805 \\
\hline \multirow[t]{5}{*}{ Chloride } & TDS & $Y=0.0526 X+12.038$ & 0.3388 & 0.4737 & 1.5263 & 0.0526 \\
\hline & Hardness & $Y=0.2962 X-23.156$ & 0.7762 & 0.3519 & 1.6481 & 0.2962 \\
\hline & Alkalinity & $Y=0.3154 X+3.7024$ & 0.3028 & 0.3423 & 1.6577 & 0.3154 \\
\hline & Sulfate & $Y=0.0532 X+6.6431$ & 0.4152 & 0.4734 & 1.5266 & 0.0532 \\
\hline & Conductance & $Y=0.072 X+10.415$ & 0.4797 & 0.464 & 1.536 & 0.072 \\
\hline \multirow[t]{5}{*}{ Conductance } & TDS & $Y=0.8297 X-21.447$ & 0.9099 & 0.08515 & 1.91485 & 0.8297 \\
\hline & Hardness & $Y=2.2467 X-96.508$ & 0.4825 & 0.62335 & 1.37665 & 0.2467 \\
\hline & Alkalinity & $Y=2.6941 X+76.75$ & 0.2388 & 0.84705 & 1.15295 & 0.6941 \\
\hline & Sulfate & $Y=0.6181 X+13.027$ & 0.606 & 0.19095 & 1.80905 & 0.6181 \\
\hline & $\mathrm{Zn}$ & $Y=2.4368 X+341.59$ & 0.0003 & 0.7184 & 1.2816 & 0.4368 \\
\hline
\end{tabular}


Table 4 continued

\begin{tabular}{|c|c|c|c|c|c|c|}
\hline$Y$ & Parameters $(X)$ & Regression equation & $r^{2}$ & $H$ & $D$ (Fractal) & PI \\
\hline \multirow[t]{15}{*}{ BOD } & $\mathrm{pH}$ & $Y=-0.154 X+5.8395$ & 0.0101 & 0.575 & 1.425 & 0.15 \\
\hline & TDS & $Y=-0.0007 X+4.8817$ & 0.1484 & 0.50035 & 1.49965 & 0.0007 \\
\hline & Hardness & $Y=-0.0038 X+5.3$ & 0.2781 & 0.5019 & 1.4981 & 0.0038 \\
\hline & Alkalinity & $Y=0.0032 X+4.2226$ & 0.0704 & 0.4984 & 1.5016 & 0.0032 \\
\hline & Sulfate & $Y=-0.0005 X+4.8404$ & 0.0922 & 0.50025 & 1.49975 & 0.0005 \\
\hline & Chloride & $Y=-0.0083 X+4.8459$ & 0.1522 & 0.50415 & 1.49585 & 0.0083 \\
\hline & Conductance & $Y=-0.0012 X+4.9607$ & 0.2819 & 0.5006 & 1.4994 & 0.0012 \\
\hline & DO & $Y=-0.7504 X+11.569$ & 0.4749 & 0.8752 & 1.1248 & 0.7504 \\
\hline & $\mathrm{Ca}$ & $Y=-0.0145 X+4.9754$ & 0.1432 & 0.50725 & 1.49275 & 0.0145 \\
\hline & $\mathrm{Fe}$ & $Y=-0.0633 X+4.5843$ & 0.0199 & 0.53165 & 1.46835 & 0.0633 \\
\hline & $\mathrm{Cr}$ & $Y=0.0438 X+4.525$ & 0.0033 & 0.4781 & 1.5219 & 0.0438 \\
\hline & $\mathrm{Zn}$ & $Y=-0.1883 X+5.0711$ & 0.4188 & 0.59415 & 1.40585 & 0.1883 \\
\hline & $\mathrm{Fl}$ & $Y=-0.2239 X+4.8392$ & 0.119 & 0.61195 & 1.38805 & 0.2239 \\
\hline & Nitrate & $Y=-0.0633 X+4.8507$ & 0.0786 & 0.53165 & 1.46835 & 0.0633 \\
\hline & Nitrite & $Y=0.2607 X+4.3336$ & 0.3118 & 0.36965 & 1.63035 & 0.2607 \\
\hline \multirow[t]{15}{*}{ DO } & $\mathrm{pH}$ & $Y=-0.6873 X+15.107$ & 0.2392 & 0.84365 & 1.15635 & 0.6873 \\
\hline & TDS & $Y=-9 \mathrm{E}-05 X+9.3937$ & 0.0025 & 0.500045 & 1.499955 & $9 \mathrm{E}-05$ \\
\hline & Hardness & $Y=-4 \mathrm{E}-05 X+9.3617$ & 0.00003 & 0.50002 & 1.49998 & $4 \mathrm{E}-05$ \\
\hline & Alkalinity & $Y=-0.0078 X+10.143$ & 0.4849 & 0.5039 & 1.4961 & 0.0078 \\
\hline & Sulfate & $Y=-0.0004 X+9.5979$ & 0.077 & 0.5002 & 1.4998 & 0.0004 \\
\hline & Chloride & $Y=-0.0056 X+9.5525$ & 0.0811 & 0.5028 & 1.4972 & 0.0056 \\
\hline & Conductance & $Y=-3 \mathrm{E}-05 X+9.3639$ & 0.0002 & 0.500015 & 1.499985 & $3 \mathrm{E}-05$ \\
\hline & BOD & $Y=-0.6329 X+12.234$ & 0.4749 & 0.81645 & 1.18355 & 0.6329 \\
\hline & $\mathrm{Ca}$ & $Y=-0.0028 X+9.4372$ & 0.0065 & 0.5014 & 1.4986 & 0.0028 \\
\hline & $\mathrm{Fe}$ & $Y=-0.1773 X+9.4501$ & 0.1851 & 0.58865 & 1.41135 & 0.1773 \\
\hline & $\mathrm{Cr}$ & $Y=0.0379 X+9.3325$ & 0.0029 & 0.48105 & 1.51895 & 0.0379 \\
\hline & $\mathrm{Zn}$ & $Y=0.182 X+8.8506$ & 0.4638 & 0.409 & 1.591 & 0.182 \\
\hline & $\mathrm{Fl}$ & $Y=0.2856 X+8.9853$ & 0.2295 & 0.3572 & 1.6428 & 0.2856 \\
\hline & Nitrate & $Y=-0.0633 X+9.6549$ & 0.0932 & 0.53165 & 1.46835 & 0.0633 \\
\hline & Nitrite & $Y=-0.4094 X+9.694$ & 0.9118 & 0.7047 & 1.2953 & 0.4094 \\
\hline \multirow[t]{10}{*}{$\mathrm{Ca}$} & TDS & $Y=0.0333 X+14.544$ & 0.4352 & 0.48335 & 1.51665 & 0.0333 \\
\hline & Hardness & $Y=0.1027 X+9.0587$ & 0.299 & 0.44865 & 1.55135 & 0.1027 \\
\hline & Alkalinity & $Y=0.1003 X+19.285$ & 0.0981 & 0.44985 & 1.55015 & 0.1003 \\
\hline & Sulfate & $Y=0.0335 X+11.206$ & 0.5288 & 0.48325 & 1.51675 & 0.0335 \\
\hline & Chloride & $Y=0.3419 X+17.258$ & 0.3748 & 0.32905 & 1.67095 & 0.3419 \\
\hline & Conductance & $Y=0.0408 X+15.188$ & 0.4932 & 0.4796 & 1.5204 & 0.0408 \\
\hline & DO & $Y=2.2894 X+50.808$ & 0.0065 & 0.6447 & 1.3553 & 0.2894 \\
\hline & $\mathrm{Cr}$ & $Y=2.522 X+27.953$ & 0.0159 & 0.761 & 1.239 & 0.522 \\
\hline & $\mathrm{Zn}$ & $Y=0.7954 X+0.746$ & 0.0101 & 0.1023 & 1.8977 & 0.7954 \\
\hline & Nitrite & $Y=1.4735 X+28.169$ & 0.0146 & 0.23675 & 1.76325 & 0.5265 \\
\hline \multirow[t]{8}{*}{$\mathrm{Fe}$} & $\mathrm{pH}$ & $Y=2.6637 X-21.756$ & 0.0103 & 0.83185 & 1.16815 & 0.6637 \\
\hline & TDS & $Y=0.001 X+0.1089$ & 0.0507 & 0.4995 & 1.5005 & 0.001 \\
\hline & Hardness & $Y=0.0076 X-0.9698$ & 0.2272 & 0.4962 & 1.5038 & 0.0076 \\
\hline & Alkalinity & $Y=0.0164 X-1.116$ & 0.3629 & 0.4918 & 1.5082 & 0.0164 \\
\hline & Sulfate & $Y=0.0025 X-0.8355$ & 0.417 & 0.49875 & 1.50125 & 0.0025 \\
\hline & Chloride & $Y=0.0329 X-0.6275$ & 0.4782 & 0.48355 & 1.51645 & 0.0329 \\
\hline & Conductance & $Y=0.007 X+0.8218$ & 0.0615 & 0.4965 & 1.5035 & 0.007 \\
\hline & BOD & $Y=-0.3145 X+1.9717$ & 0.0199 & 0.65725 & 1.34275 & 0.3145 \\
\hline
\end{tabular}


Table 4 continued

\begin{tabular}{|c|c|c|c|c|c|c|}
\hline$Y$ & Parameters $(X)$ & Regression equation & $r^{2}$ & $H$ & $D$ (Fractal) & PI \\
\hline & $\mathrm{Ca}$ & $Y=0.0063 X+0.3853$ & 0.0159 & 0.49685 & 1.50315 & 0.0063 \\
\hline & $\mathrm{Cr}$ & $Y=-0.7365 X+0.9613$ & 0.1869 & 0.86825 & 1.13175 & 0.7365 \\
\hline & $\mathrm{Zn}$ & $Y=0.0741 X+0.746$ & 0.0131 & 0.46295 & 1.53705 & 0.0741 \\
\hline & $\mathrm{Fl}$ & $Y=-0.4372 X+1.1055$ & 0.0914 & 0.7186 & 1.2814 & 0.4372 \\
\hline & Nitrate & $Y=0.3799 X-1.2631$ & 0.5695 & 0.31005 & 1.68995 & 0.3799 \\
\hline & Nitrite & $Y=0.591 X+0.0503$ & 0.3228 & 0.2045 & 1.7955 & 0.591 \\
\hline \multirow[t]{15}{*}{$\mathrm{Cr}$} & $\mathrm{pH}$ & $Y=-0.4665 X+4.4758$ & 0.0543 & 0.73325 & 1.26675 & 0.4665 \\
\hline & TDS & $Y=-0.0002 X+0.6687$ & 0.0076 & 0.5001 & 1.4999 & 0.0002 \\
\hline & Hardness & $Y=-0.0019 X+0.9488$ & 0.0413 & 0.50095 & 1.49905 & 0.0019 \\
\hline & Alkalinity & $Y=-0.0047 X+1.0489$ & 0.0877 & 0.50235 & 1.49765 & 0.0047 \\
\hline & Sulfate & $Y=-7 \mathrm{E}-05 X+0.6065$ & 0.0018 & 0.500035 & 1.499965 & $7 \mathrm{E}-05$ \\
\hline & Chloride & $Y=-0.0079 X+0.8499$ & 0.0792 & 0.50395 & 1.49605 & 0.0079 \\
\hline & Conductance & $Y=0.002 X-0.1531$ & 0.1621 & 0.499 & 1.501 & 0.002 \\
\hline & BOD & $Y=0.075 X+0.2296$ & 0.0033 & 0.4625 & 1.5375 & 0.075 \\
\hline & DO & $Y=0.0769 X-0.1489$ & 0.0029 & 0.46155 & 1.53845 & 0.0769 \\
\hline & $\mathrm{Ca}$ & $Y=0.0429 X-0.721$ & 0.2538 & 0.47855 & 1.52145 & 0.0429 \\
\hline & $\mathrm{Fe}$ & $Y=-0.2538 X+0.7081$ & 0.1869 & 0.6269 & 1.3731 & 0.2538 \\
\hline & $\mathrm{Zn}$ & $Y=-0.0169 X+0.6175$ & 0.002 & 0.50845 & 1.49155 & 0.0169 \\
\hline & $\mathrm{Fl}$ & $Y=0.4731 X-0.0402$ & 0.3106 & 0.26345 & 1.73655 & 0.4731 \\
\hline & Nitrate & $Y=-0.0625 X+0.8675$ & 0.0447 & 0.53125 & 1.46875 & 0.0625 \\
\hline & Nitrite & $Y=-0.0826 X+0.6394$ & 0.0183 & 0.5413 & 1.4587 & 0.0826 \\
\hline \multirow[t]{13}{*}{$\mathrm{Zn}$} & TDS & $Y=-0.001 X+3.2144$ & 0.0229 & 0.5005 & 1.4995 & 0.001 \\
\hline & Hardness & $Y=-0.0031 X+3.3903$ & 0.0163 & 0.50155 & 1.49845 & 0.0031 \\
\hline & Alkalinity & $Y=-0.0269 X+5.4753$ & 0.408 & 0.51345 & 1.48655 & 0.0269 \\
\hline & Sulfate & $Y=-0.0012 X+3.4082$ & 0.0381 & 0.5006 & 1.4994 & 0.0012 \\
\hline & Chloride & $Y=-0.0172 X+3.3756$ & 0.0546 & 0.5086 & 1.4914 & 0.0172 \\
\hline & Conductance & $Y=0.0001 X+2.7172$ & 0.0003 & 0.49995 & 1.50005 & $1 \mathrm{E}-04$ \\
\hline & DO & $Y=2.5482 X-21.069$ & 0.4638 & 0.7741 & 1.2259 & 0.5482 \\
\hline & $\mathrm{Ca}$ & $Y=-0.0132 X+3.1551$ & 0.0101 & 0.5066 & 1.4934 & 0.0132 \\
\hline & $\mathrm{Fe}$ & $Y=-0.176 X+2.862$ & 0.0131 & 0.588 & 1.412 & 0.176 \\
\hline & $\mathrm{Cr}$ & $Y=-0.1164 X+2.8331$ & 0.002 & 0.5582 & 1.4418 & 0.1164 \\
\hline & $\mathrm{Fl}$ & $Y=0.03721 X+2.2861$ & 0.0278 & 0.481395 & 1.518605 & 0.03721 \\
\hline & Nitrate & $Y=-0.02104 X+3.7659$ & 0.0735 & 0.51052 & 1.48948 & 0.02104 \\
\hline & Nitrite & $Y=-0.8867 X+3.5026$ & 0.3055 & 0.94335 & 1.05665 & 0.8867 \\
\hline \multirow[t]{15}{*}{$\mathrm{Fl}$} & $\mathrm{pH}$ & $Y=0.1176 X+0.304$ & 0.0025 & 0.4412 & 1.5588 & 0.1176 \\
\hline & TDS & $Y=0.0012 X+0.7569$ & 0.1626 & 0.4994 & 1.5006 & 0.0012 \\
\hline & Hardness & $Y=0.0013 X+1.0398$ & 0.0132 & 0.49935 & 1.50065 & 0.0013 \\
\hline & Alkalinity & $Y=-0.0033 X+1.6288$ & 0.0314 & 0.50165 & 1.49835 & 0.0033 \\
\hline & Sulfate & $Y=0.0008 X+0.8503$ & 0.0897 & 0.4996 & 1.5004 & 0.0008 \\
\hline & Chloride & $Y=-0.0042 X+1.4398$ & 0.0161 & 0.5021 & 1.4979 & 0.0042 \\
\hline & Conductance & $Y=0.009 X+0.9714$ & 0.0722 & 0.4955 & 1.5045 & 0.009 \\
\hline & BOD & $Y=1.1961 X-4.612$ & 0.3138 & 0.09805 & 1.90195 & 0.8039 \\
\hline & $\mathrm{DO}$ & $Y=0.8038 X-6.227$ & 0.2295 & 0.0981 & 1.9019 & 0.8038 \\
\hline & $\mathrm{Ca}$ & $Y=0.0239 X+0.5901$ & 0.1641 & 0.48805 & 1.51195 & 0.0239 \\
\hline & $\mathrm{Fe}$ & $Y=-0.209 X+1.4047$ & 0.0914 & 0.6045 & 1.3955 & 0.209 \\
\hline & $\mathrm{Cr}$ & $Y=0.6565 X+0.9169$ & 0.3106 & 0.17175 & 1.82825 & 0.6565 \\
\hline & $\mathrm{Zn}$ & $Y=0.074 X+1.0847$ & 0.0278 & 0.463 & 1.537 & 0.074 \\
\hline & Nitrate & $Y=0.0594 X+1.0096$ & 0.0291 & 0.4703 & 1.5297 & 0.0594 \\
\hline & Nitrite & $Y=-0.3392 X+1.5732$ & 0.2224 & 0.6696 & 1.3304 & 0.3392 \\
\hline
\end{tabular}


Table 4 continued

\begin{tabular}{|c|c|c|c|c|c|c|}
\hline$Y$ & Parameters $(X)$ & Regression equation & $r^{2}$ & $H$ & $D$ (Fractal) & PI \\
\hline \multirow[t]{12}{*}{ Nitrate } & TDS & $Y=0.0066 X+1.0845$ & 0.5969 & 0.4967 & 1.5033 & 0.0066 \\
\hline & Hardness & $Y=0.021 X+0.5819$ & 0.4381 & 0.4895 & 1.5105 & 0.021 \\
\hline & Alkalinity & $Y=0.0404 X+0.6736$ & 0.5564 & 0.4798 & 1.5202 & 0.0404 \\
\hline & Sulfate & $Y=0.0073 X+0.8027$ & 0.8686 & 0.49635 & 1.50365 & 0.0073 \\
\hline & Chloride & $Y=0.0712 X+2.2206$ & 0.5672 & 0.4644 & 1.5356 & 0.0712 \\
\hline & Conductance & $Y=0.008 X+1.9559$ & 0.6652 & 0.496 & 1.504 & 0.008 \\
\hline & $\mathrm{Ca}$ & $Y=0.1323 X+0.8597$ & 0.6109 & 0.43385 & 1.56615 & 0.1323 \\
\hline & $\mathrm{Fe}$ & $Y=1.4989 X+3.9377$ & 0.5695 & 0.24945 & 1.75055 & 0.5011 \\
\hline & $\mathrm{Cr}$ & $Y=-0.7154 X+5.1567$ & 0.0447 & 0.8577 & 1.1423 & 0.7154 \\
\hline & $\mathrm{Zn}$ & $Y=-0.3492 X+5.7144$ & 0.0735 & 0.6746 & 1.3254 & 0.3492 \\
\hline & $\mathrm{Fl}$ & $Y=0.4902 X+4.1152$ & 0.0291 & 0.2549 & 1.7451 & 0.4902 \\
\hline & Nitrite & $Y=0.8566 X+4.0374$ & 0.1718 & 0.0717 & 1.9283 & 0.8566 \\
\hline \multirow[t]{14}{*}{ Nitrite } & $\mathrm{pH}$ & $Y=2.0118 X-16.011$ & 0.3767 & 0.5059 & 1.4941 & 0.0118 \\
\hline & TDS & $Y=0.0005 X+0.6238$ & 0.0125 & 0.49975 & 1.50025 & 0.0005 \\
\hline & Hardness & $Y=0.0024 X+0.3561$ & 0.0242 & 0.4988 & 1.5012 & 0.0024 \\
\hline & Alkalinity & $Y=0.0185 X-1.031$ & 0.4956 & 0.49075 & 1.50925 & 0.0185 \\
\hline & Sulfate & $Y=0.0015 X+0.0206$ & 0.1561 & 0.49925 & 1.50075 & 0.0015 \\
\hline & Chloride & $Y=0.0177 X+0.2025$ & 0.1492 & 0.49115 & 1.50885 & 0.0177 \\
\hline & Conductance & $Y=0.0006 X+0.6311$ & 0.0144 & 0.4997 & 1.5003 & 0.0006 \\
\hline & BOD & $Y=-0.5316 X+3.7104$ & 0.119 & 0.7658 & 1.2342 & 0.5316 \\
\hline & $\mathrm{Ca}$ & $Y=0.0099 X+0.5394$ & 0.0146 & 0.49505 & 1.50495 & 0.0099 \\
\hline & $\mathrm{Fe}$ & $Y=0.5461 X+0.5346$ & 0.3228 & 0.22695 & 1.77305 & 0.5461 \\
\hline & $\mathrm{Cr}$ & $Y=-0.217 X+0.9565$ & 0.0183 & 0.6085 & 1.3915 & 0.217 \\
\hline & $\mathrm{Zn}$ & $Y=-0.3446 X+1.7833$ & 0.3055 & 0.6723 & 1.3277 & 0.3446 \\
\hline & $\mathrm{Fl}$ & $Y=-0.6556 X+1.6769$ & 0.2224 & 0.8278 & 1.1722 & 0.6556 \\
\hline & Nitrate & $Y=0.2006 X-0.1226$ & 0.1718 & 0.3997 & 1.6003 & 0.2006 \\
\hline
\end{tabular}

behavior with TDS, hardness, alkalinity, sulfate, chloride, conductance and $\mathrm{Ca}$ parameters. DO has persistent behavior with $\mathrm{pH}, \mathrm{BOD}, \mathrm{Fe}$, nitrate and nitrite and antipersistent behavior with $\mathrm{Cr}, \mathrm{Zn}$ and $\mathrm{Fl}$ parameters.

\section{$\mathrm{Ca}$}

Mean, median and mode values are almost same and thus the curve shows normal behavior. Standard deviation value is high (4.86); thus, the values of $\mathrm{Ca}$ are not close to each other. It is negatively skewed and the curve is platykurtic. $\mathrm{Ca}$ has persistent behavior with $\mathrm{DO}$ and $\mathrm{Cr}$, and anti-persistent behavior with TDS, hardness, alkalinity, sulfate, chloride, conductance, $\mathrm{Zn}$ and nitrite parameters.

$\mathrm{Fe}$

Average, median and mode values are approximately equal and thus the data show normal behavior. Standard deviation value (0.414) exhibits that the sample points are close to each other. Skewness value suggests that the curve is symmetrical and kurtosis value is less than 3; thus, the curve is platykurtic. Fe has Brownian time series (True random walk) behavior with TDS, hardness, alkalinity, sulfate, conductance and $\mathrm{Ca}$ parameters. Fe has persistent behavior with $\mathrm{pH}, \mathrm{BOD}, \mathrm{Cr}$ and $\mathrm{Fl}$, and anti-persistent behavior with chloride, $\mathrm{Zn}$, nitrate and nitrite parameters.

$\mathrm{Cr}$

Average, median and mode values are the same and thus data show normal behavior. Standard deviation value (0.243) explains that the sample data are not spread. The curve is negatively skewed and platykurtic. $\mathrm{Cr}$ has Brownian time series (True random walk) behavior with TDS, hardness, alkalinity, sulfate, chloride, conductance and $\mathrm{Zn}$ parameters. $\mathrm{Cr}$ has persistent behavior with $\mathrm{pH}, \mathrm{Fe}$, nitrate, nitrite, and anti-persistent behavior with BOD, DO, $\mathrm{Ca}$ and $\mathrm{Fl}$ parameters.

\section{$\mathrm{Zn}$}

Mean and median values are approximately equal. Standard deviation (0.639) suggests that the sample data are 
very close to each other. The skewness value is 1.485 , and thus the curve is not symmetrical and platykurtic. $\mathrm{Zn}$ has Brownian time series (True random walk) behavior with TDS, Hardness, sulfate, chloride, conductance and $\mathrm{Ca}$ parameters. Zn has persistent behavior with alkalinity, DO, $\mathrm{Fe}, \mathrm{Cr}$, nitrate and nitrite, and anti-persistent behavior with Fl parameter.

Fl

Mean, median and mode values are approximately equal, and thus the curve shows normal behavior. Standard deviation value (0.287) suggests that the sample data are close to each other and the curve is platykurtic. Fl has Brownian time series (true random walk) behavior with TDS, hardness, alkalinity, sulfate, chloride and conductance parameters. $\mathrm{Fl}$ has persistent behavior with $\mathrm{Fe}$ and nitrite, and anti-persistent behavior with BOD, DO, Ca, Cr, $\mathrm{Zn}$ and nitrate parameters.

\section{Nitrate}

Average, median and mode values are equal and thus data exhibit normal behavior. Standard deviation (0.823) suggests that the data are close to each other. The skewness value $(-0.032)$ is approximately equal to 0 and thus the curve is symmetrical and platykurtic. Nitrate has Brownian time series (true random walk) behavior with TDS, sulfate and conductance parameters. Nitrate has persistent behavior with $\mathrm{Cr}$ and $\mathrm{Zn}$, and anti-persistent behavior with hardness, alkalinity, chloride, $\mathrm{Ca}, \mathrm{Fe}, \mathrm{Fl}$ and nitrite parameters.

\section{Nitrite}

Mean, median and mode values are approximately equal, so it is symmetrical. Standard deviation (0.398) suggests that the sample data are close to each other. The skewness value is approximately equal to 0 , and thus the curve is symmetrical and platykurtic. Nitrite has Brownian time series (true random walk) behavior with $\mathrm{pH}$, TDS, hardness, alkalinity, sulfate, chloride, conductance and $\mathrm{Ca}$ parameters. Nitrite has persistent behavior with BOD, $\mathrm{Cr}$, $\mathrm{Zn}$ and $\mathrm{Fl}$, and anti-persistent behavior with $\mathrm{Fe}$ and nitrate parameters.

\section{Conclusion}

It has been observed that all water parameters at the confluence of Beas and Sutlej rivers at Harike Lake have platykurtic curve. The Brownian time series behavior exists for $\mathrm{pH}$ with TDS, hardness, alkalinity, sulfate, chloride and conductance parameters; BOD with TDS, hardness, alkalinity, sulfate, chloride, conductance and Ca parameters; DO with TDS, hardness, alkalinity, sulfate, chloride, conductance and Ca parameters; Fe with TDS, hardness, alkalinity, sulfate, conductance and $\mathrm{Ca}$ parameters; $\mathrm{Cr}$ with TDS, hardness, alkalinity, sulfate, chloride, conductance and $\mathrm{Zn}$ parameters; $\mathrm{Zn}$ with TDS, hardness, sulfate, chloride, conductance and $\mathrm{Ca}$ parameters; Fl with TDS, hardness, alkalinity, sulfate, chloride and conductance parameters; nitrate with TDS, sulfate and conductance parameters; nitrite with $\mathrm{pH}$, TDS, hardness, alkalinity, sulfate, chloride, conductance, and $\mathrm{Ca}$ parameters. The persistent behavior exists of $\mathrm{pH}$ with $\mathrm{BOD}, \mathrm{DO}, \mathrm{Cr}$ and $\mathrm{Zn}$; TDS with hardness; hardness with chloride, DO and $\mathrm{Ca}$; sulfate with hardness; conductance with hardness, alkalinity and $\mathrm{Zn}$; $\mathrm{BOD}$ with $\mathrm{pH}, \mathrm{DO}, \mathrm{Fe}, \mathrm{Zn}, \mathrm{Fl}$ and nitrate; $\mathrm{DO}$ with $\mathrm{pH}, \mathrm{BOD}, \mathrm{Fe}$, nitrate and nitrite; $\mathrm{Ca}$ with $\mathrm{DO}$ and $\mathrm{Cr}$; $\mathrm{Fe}$ with $\mathrm{pH}, \mathrm{BOD}, \mathrm{Cr}$ and $\mathrm{Fl}$; $\mathrm{Cr}$ with $\mathrm{pH}, \mathrm{Fe}$, nitrate and nitrite; $\mathrm{Zn}$ with alkalinity, $\mathrm{DO}, \mathrm{Fe}, \mathrm{Cr}$, nitrate and nitrite; $\mathrm{Fl}$ with $\mathrm{Fe}$ and nitrite; nitrate with $\mathrm{Cr}$ and $\mathrm{Zn}$; nitrite has persistent behavior with BOD, $\mathrm{Cr}, \mathrm{Zn}$ and Fl. The antipersistent behavior exists of $\mathrm{pH}$ with $\mathrm{Ca}, \mathrm{Fe}, \mathrm{Fl}$, nitrate and nitrite; TDS with sulfate and conductance; hardness with TDS, alkalinity, sulfate and conductance; alkalinity with TDS, hardness, sulfate, chloride, conductance and $\mathrm{Ca}$; sulfate with TDS and conductance; chloride with TDS, hardness, alkalinity, sulfate and conductance; conductance with TDS and sulfate; BOD with $\mathrm{Cr}$ and nitrite; DO with $\mathrm{Cr}, \mathrm{Zn}$ and $\mathrm{Fl}$; Ca with TDS, hardness, alkalinity, sulfate, chloride, conductance, $\mathrm{Zn}$ and nitrite; Fe with chloride, $\mathrm{Zn}$, nitrate and nitrite; $\mathrm{Cr}$ with $\mathrm{BOD}, \mathrm{DO}, \mathrm{Ca}$ and $\mathrm{Fl}$; $\mathrm{Zn}$ with $\mathrm{Fl}$; Fl with BOD, DO, Ca, Cr, Zn and nitrate; nitrate with hardness, alkalinity, chloride, $\mathrm{Ca}, \mathrm{Fe}, \mathrm{Fl}$ and nitrite; nitrite with $\mathrm{Fe}$ and nitrate parameters. The parameters $\mathrm{pH}$, DO, hardness, $\mathrm{Cr}$ and sulfate crossed the prescribed WHO/EPA standard values for all months. Thus, the water of Harike Lake is severely contaminated and is not fit for drinking and industrial purpose.

Acknowledgments The authors are thankful to Guru Gobind Singh, Indraprastha University, Delhi (India) for providing research facilities and financial support (F.IPU/GGSIPU/USBAS/Ph.D./2010/452 dated $15 / 04 / 10)$.

\section{References}

Akoto O, Adiyiah J (2007) Chemical analysis of drinking water from some communities in the Brong Ahafo region. Int J Environ Sci Technol 4(2):211-214

Aksoy H, Toprak ZF, Aytek A, Ünal NE (2004) Stochastic generation of hourly mean wind speed data. Renew Energy 29(14):2111-2131

Alam JB, Muyen Z, Islam MR, Islam S, Mamun M (2007) Water quality parameters along rivers. Int J Environ Sci Tech 4(1):159-167

APHA (1995) Standard methods for examination of water and waste water. 19th edn, American Public Health Association, Washington D.C. 
Banu JR, Kaliappan S, Yeom IT (2007) Treatment of domestic wastewater using upflow anaerobic sludge blanket reactor. Int J Environ Sci Technol 4(3):363-370

Carlson E, Ecker MD (2002) A statistical examination of water quality in two Iowa Lakes. Am J Undergrad Res 1(2):31-45

Chenini I, Khemiri S (2009) Evaluation of ground water quality using multiple linear regression and structural equation modeling. Int $\mathbf{J}$ Environ Sci Technol 6(3):509-519

Chenini I, Ben Mammou A, Turki MM (2008) Groundwater resources of a multi-layered aquiferous system in arid area: data analysis and water budgeting. Int J Environ Sci Technol 5(3):361-374

Imo TS, Oomori T, Toshihiko M, Tamaki F (2007) The comparative study of trihalomethanes in drinking waters. Int J Environ Sci Technol 4(4):421-426

Jain P, Sharma JD, Sohu D, Sharma P (2005) Chemical analysis of drinking water of villages of Sanganer Tehsil, Jaipur District. Int J Environ Sci Technol 2(4):373-379

Joarder MAM, Raihan F, Alam JB, Hasanuzzaman S (2008) Regression analysis of ground water quality data of Sunamganj District, Bangladesh. Int J Environ Res 2(3):291-296

Juang DF, Tsai WP, Liu WK, Lin JH (2008) Treatment of polluted river water by a gravel contact oxidation system constructed under riverbed. Int J Environ Sci Technol 5(3):305-314

Kahya E, Kalayci S (2004) Trend analysis of streamflow in Turkey. J Hydrol 289:128-144

Korashey R (2009) Using regression analysis to estimate water quality constituents in Bahr El Baqar drain. J Appl Sci Res 5(8):1067-1076

Kumar A, Dua A (2009) Water quality index for assessment of water quality of river Ravi at Modhopur (India). G J Environ Sci $8(1): 49-57$

Mousavi M, Kiani S, Lotfi S, Naeemi N, Honarmand M (2008) Transient and spatial modelling and simulation of polybrominated diphenyl ethers reaction and transport in air, water and soil. Int J Environ Sci Technol 5(3):323-330

Movahed M, Hermanisc E (2008) Fractal analysis of river flow fluctuations. Phys A 387:915-932

Park J, Park C (2009) Robust estimation of the Hurst parameter and selection of an onset scaling. Stat Sinica 19:1531-1555

Parmar KS, Chugh P, Minhas P, Sahota HS (2009) Alarming pollution levels in rivers of Punjab. Indian $\mathrm{J}$ Environ Prot 29(11):953-959

Phiri O, Mumba P, Moyo BHZ, Kadewa W (2005) Assessment of the impact of industrial effluents on water quality of receiving rivers in urban areas of Malawi. Int $\mathrm{J}$ Environ Sci Technol 2(3):237-244

Prasad BG, Narayana TS (2004) Subsurface water quality of different sampling stations with some selected parameters at Machilipatnam Town. Nat Environ Pollut Tech 3(1):47-50

Psargaonkar A, Gupta A, Devotta S (2008) Multivariate analysis of ground water resources in Ganga-Yamuna Basin(India). J Environ Sci Eng 50(3):215-222

Rangarajan G (1997) A climate predictability index and its applications. Geophys Res Lett 24(10):1239-1242

Rangarajan G, Ding M (2000) Integrated approach to the assessment of long range correlation in time series data. Phys Rev E 61(5):4991-5001

Rangarajan G, Sant DA (2004) Fractal dimensional analysis of Indian climatic dynamics. Chaos, Solitons Fractals 19:285-291

Shukla JB, Misra AK, Chandra P (2008) Mathematical modeling and analysis of the depletion of dissolved oxygen in eutrophied water bodies affected by organic pollutant. Non-Linear Anal Real world Appl 9:1851-1865

Sinha DK, Kumar N (2010) Drinking water quality management through correlation studies among various physico-chemical parameters: a case study. Int. J Environ Sci 1(2):253-259

Toprak ZF (2009) Flow discharge modeling in open canals using a new fuzzy modeling technique (SMRGT). CLEAN-Soil Air Water 37(9):742-752

Toprak ZF, Cigizoglu HK (2008) Predicting longitudinal dispersion coefficient in natural streams by artificial intelligence methods. Hydrol Process 22(20):4106-4129

Toprak ZF, Savci ME (2007) Longitudinal dispersion coefficient modeling in natural channels using fuzzy logic. CLEAN-Soil Air Water 35(6):626-637

Toprak ZF, Sen Z, Savci ME (2004) Comment on Longitudinal dispersion coefficients in natural channels. Water Res 38(13): 3139-3143

Toprak ZF, Eris E, Agiralioglu N, Cigizoglu HK, Yilmaz L, Aksoy H, Coskun G, Andic G, Alganci U (2009) Modeling monthly mean flow in a poorly gauged basin by fuzzy logic. CLEAN-Soil Air Water 37(7):555-564

Vassilis Z, Antonopoulos M, Mitsiou AK (2001) Statistical and trend analysis of water quality and quantity data for the Strymon River in Greece. Hydrol Earth Syst Sci 5(4):679-691

WHO (1971) International standards for drinking water. World Health Organization, Geneva 